\title{
Radiomics Analysis of Contrast-enhanced CT for Staging Liver Fibrosis: An Update for Image Biomarker
}

Jin-Cheng Wang ( $\sim 1057770573 @ q q . c o m)$

Nanjing Medical University https://orcid.org/0000-0001-9345-2388

Shengnan Tang

Nanjing Drum Tower Hospital: Nanjing University Medical School Affiliated Nanjing Drum Tower Hospital

Yingfan Mao

Nanjing Drum Tower Hospital: Nanjing University Medical School Affiliated Nanjing Drum Tower Hospital

Jin Wu

Nanjing Drum Tower Hospital: Nanjing University Medical School Affiliated Nanjing Drum Tower Hospital

\section{Shanshan Xu}

Nanjing Drum Tower Hospital: Nanjing University Medical School Affiliated Nanjing Drum Tower Hospital

\section{Qi Yue}

Nanjing Drum Tower Hospital: Nanjing University Medical School Affiliated Nanjing Drum Tower Hospital

\section{Jun Chen}

Nanjing Drum Tower Hospital: Nanjing University Medical School Affiliated Nanjing Drum Tower Hospital

\section{Jian He}

Nanjing Drum Tower Hospital: Nanjing University Medical School Affiliated Nanjing Drum Tower Hospital

\section{Yin Yin}

Nanjing Drum Tower Hospital: Nanjing University Medical School Affiliated Nanjing Drum Tower Hospital

\section{Research}

Keywords: radiomics, contrast-enhanced CT, liver fibrosis, prediction model 
Posted Date: October 25th, 2021

DOl: https://doi.org/10.21203/rs.3.rs-987914/v1

License: (c) (i) This work is licensed under a Creative Commons Attribution 4.0 International License. Read Full License

Version of Record: A version of this preprint was published at Hepatology International on March 28th, 2022. See the published version at https://doi.org/10.1007/s12072-022-10326-7. 


\section{Abstract}

\section{Background}

To establish and validate a radiomics-based model for staging liver fibrosis at contrast-enhanced CT images.

\section{Materials and Methods}

This retrospective study developed two radiomics-based models (R-score: radiomics signature; R-fibrosis: integrate radiomic and serum variables) in a training cohort of 332 patients (median age, 59 years; interquartile range, 51-67 years; 256 men) with biopsy-proven liver fibrosis who underwent contrastenhanced CT between January 2017 and December 2020. Radiomic features were extracted from noncontrast, arterial and portal phase CT images and selected by using the least absolute shrinkage and selection operator (LASSO) logistic regression to differentiate stage F3-F4 from stage F0-F2. Optimal cutoffs to diagnose significant fibrosis (stage F2-F4), advanced fibrosis (stage F3-F4) and cirrhosis (stage F4) were determined by receiver operating characteristic curve analysis. Diagnostic performance was evaluated by area under the curve, Obuchowski index, calibrations and decision curve analysis. An internal validation was conducted in 111 randomly assigned patients (median age, 58 years; interquartile range, 49-66 years; 89 men).

Results

In the validation cohort, R-score and R-fibrosis (Obuchowski index, 0.843 and 0.846 , respectively) significantly outperformed aspartate transaminase-to-platelet ratio (APRI) (Obuchowski index, 0.651; $P<$ .001 ) and fibrosis-4 index (FIB-4) (Obuchowski index, 0.676; $P<.001$ ) for staging liver fibrosis. By using the cutoffs, R-fibrosis and R-score had a sensitivity range of $70 \%-87 \%$, specificity range of $71 \%-97 \%$, and accuracy range of $82 \%-86 \%$ in diagnosing significant fibrosis, advanced fibrosis and cirrhosis.

\section{Conclusion}

Radiomic analysis of contrast-enhanced CT images can reach great diagnostic performance of liver fibrosis.

\section{Introduction}

Liver fibrosis is an important cause of morbidity and mortality in patients with chronic insults (e.g. viral hepatitis, alcohol and non-alcoholic fatty liver diseases [NAFLD]) and complications mainly occur in advanced fibrosis ${ }^{[1]}$. Fibrosis staging is an essential step in the clinical assessment of patients with chronic liver disease to identify those who require treatment ${ }^{[2]}$. Liver biopsy is the current reference method for staging fibrosis, but it has defects including invasiveness, sample biases and interobserver variability ${ }^{[3-6]}$. Therefore, there is a need for noninvasive and accurate methods for staging liver fibrosis. 
2018 practice guidance of the American Association for the Study of Liver Diseases (AASLD) recommended multiphase CT or MRI for initial diagnostic testing in at-risk patients with abnormal surveillance test results ${ }^{[7]}$. Compared to MRI, CT offers unique advantages including low cost, fewer contradictions, nearly ubiquitous availability and whole organ imaging capacity ${ }^{[8]}$. To date, several studies have evaluated the ability of contrast-enhanced CT imaging to determine the severity of liver fibrosis ${ }^{[8-10]}$. However, the sample sizes of these studies were not big enough and that may not be sufficient for development and validation of models.

In the era of personalized medicine, radiomics has allowed large number of quantitative features to be extracted from images that provide information on shape, signal intensity and texture ${ }^{[11,12]}$. Our previous study established and validated a radiomics-based model at non-contrast CT for the prediction of cirrhosis in patients with hepatitis $B$ virus (HBV) ${ }^{[13]}$. We hypothesized that a model based on radiomics features extracted from contrast-enhanced CT images may improve the staging of liver fibrosis. Therefore, the aim of this study was to develop and validate a radiomics model for the prediction of liver fibrosis by using contrast-enhanced CT in the liver.

\section{Materials And Methods}

This retrospective study was approved by the institutional review board of our institution, and the requirement for written informed consent was waived.

\section{Patients}

Among the 1779 consecutive patients who underwent abdominal contrast-enhanced CT at our institution between January 2017 and December 2020, patients over 18 years who had available pathologic records of liver fibrosis within 3 months of liver images at $1.5 \mathrm{~mm}$ thickness were retrospectively reviewed (Figure 2). Of 927 eligible patients, 484 were excluded due to conditions that may interfere with the extraction of radiomic features of their nontumorous right hepatic lobes, including large $(\geq 10 \mathrm{~cm})$ or multiple $(\geq 5)$ hepatic masses $(n=276)$, a tumor thrombus in the portal vein larger than the segmental branch $(n=63)$, bile duct obstruction $(n=29)$, previous surgical resection on the right hepatic lobe $(n=28)$, poor image quality because of metal or respiratory motion artifacts $(n=60)$ and incomplete clinical data $(n=28)$.

A total of 443 patients, including 345 men (median age, 56 years; age range, 28-86 years) and 98 women (median age, 61 years; age range, 40-84 years), were finally included in this study cohort. This cohort was randomized in a three-to-one ratio into training and validation cohorts, respectively, by using computergenerated random numbers without matching of any patient characteristics.

332 patients (median age, 59 years; age range, 28-86 years; 256 men [median age, 58 years; age range, 28-86 years] and 76 women [median age, 63 years; age range, 47-84 years]) were included in the training cohort and 111 (median age, 56 years; age range, 35-75 years; 89 men [median age, 56 years; age range, 35-75 years] and 22 women [median age, 55 years; age range, 40-68 years]) were in the validation cohort. 
The flow diagram for the study population is shown in Figure 1 and Table 1 shows the demographic and clinical characteristics of the cohorts. The median interval between CT images and pathologic evaluation was 15 days \pm 18 (standard deviation; range, 1-76 days). 
Table 1

Patient Characteristics

\begin{tabular}{|c|c|c|c|}
\hline Parameter & Development $(n=332)$ & Validation $(n=111)$ & $P$ value \\
\hline Sex & & & .60 \\
\hline No. of men & $256(77.1)$ & $89(80.2)$ & \\
\hline No. of women & $76(22.9)$ & $22(19.8)$ & \\
\hline Age $(y)^{\star}$ & $59(51-67)$ & $58(49-66)$ & .49 \\
\hline Men & $57(50-66)$ & $58(49-66)$ & \\
\hline Women & $63(56-69)$ & $56(54-59)$ & \\
\hline Underlying liver disease & & & .19 \\
\hline Hepatitis B & $176(53.0)$ & $70(63.1)$ & \\
\hline Hepatitis C & $13(3.9)$ & $6(5.4)$ & \\
\hline NAFLD & $11(3.3)$ & $2(1.8)$ & \\
\hline Primary biliary cirrhosis & $6(1.8)$ & $0(0)$ & \\
\hline None & $126(38.0)$ & $33(29.7)$ & \\
\hline Hepatic tumor & & & .84 \\
\hline $\mathrm{HCC}$ & $121(36.5)$ & $41(36.9)$ & \\
\hline Other malignancy & $21(6.3)$ & $5(4.5)$ & \\
\hline Hemangioma & $66(19.9)$ & $20(18.0)$ & \\
\hline None & $124(37.3)$ & $45(40.6)$ & \\
\hline Pathologic confirmation method & & & .54 \\
\hline Percutaneous liver biopsy & $74(22.3)$ & $29(26.1)$ & \\
\hline Liver resection & $232(69.9)$ & $76(68.5)$ & \\
\hline Liver transplantation & $26(7.8)$ & $6(5.4)$ & \\
\hline \multicolumn{4}{|l|}{ Laboratory findings* } \\
\hline AST (IU/mL) & $29.8(20.9-39.3)$ & $29.2(21.6-46.6)$ & .26 \\
\hline
\end{tabular}

Note. -Except where indicated, data are numbers of patients, with percentages in parentheses. ALT = alanine transferase, APRI = aspartate transaminase-to-platelet ratio, AST = aspartate transaminase, FIB-4 = fibrosis-4 index, HCC, hepatocellular carcinoma, INR = international normalized ratio, NAFLD, non-alcoholic fatty liver diseases.

*Data are medians, with interquartile range in parentheses. 


\begin{tabular}{|llll|}
\hline Parameter & Development $(\mathbf{n}=\mathbf{3 3 2})$ & Validation $(\mathbf{n}=\mathbf{1 1 1})$ & Pvalue \\
\hline ALT $(\mathrm{IU} / \mathrm{mL})$ & $27.3(19.3-45.2)$ & $32.1(21.2-49.5)$ & .16 \\
\hline Total bilirubin $(\mathrm{ng} / \mathrm{mL})$ & $12.7(9.5-17.8)$ & $13.7(9.7-17.6)$ & .42 \\
\hline Platelet count $\left(10^{9} / \mathrm{L}\right)$ & $138(95-184)$ & $143(87-180)$ & .91 \\
\hline INR & $1.03(0.97-1.10)$ & $1.01(0.96-1.08)$ & .31 \\
\hline APRI & $0.6(0.3-1.0)$ & $0.6(0.3-1.3)$ & .30 \\
\hline FIB-4 & $2.4(1.6-4.1)$ & $2.7(1.7-4.4)$ & .96 \\
\hline Metavir fibrosis stage & & & .10 \\
\hline F0 & $43(13.0)$ & $10(9.0)$ & \\
\hline F1 & $60(18.1)$ & $14(12.6)$ & \\
\hline F2 & $40(12.0)$ & $24(21.6)$ & \\
\hline F3 & $56(16.8)$ & $43(38.8)$ & \\
\hline F4 & $133(40.1)$ & & \\
\hline $\begin{array}{l}\text { Note. }- \text { Except where indicated, data are numbers of patients, with percentages in parentheses. ALT }= \\
\text { alanine transferase, APRI = aspartate transaminase-to-platelet ratio, AST = aspartate transaminase, }\end{array}$ \\
$\begin{array}{l}\text { FIB-4 = fibrosis-4 index, HCC, hepatocellular carcinoma, INR = international normalized ratio, NAFLD, } \\
\text { non-alcoholic fatty liver diseases. }\end{array}$ \\
\hline *Data are medians, with interquartile range in parentheses. \\
\hline
\end{tabular}

\section{Reference Standard for Liver Fibrosis}

Liver pathologic examination served as the reference standard for staging liver fibrosis. Liver specimens were obtained by liver resection ( $n=308)$; liver transplantation $(n=32)$; or percutaneous liver biopsy $(n=$ 103) (Table 1), which were histologically analyzed by two pathologists in consensus. Fibrosis stage was determined according to the Metavir scoring system ${ }^{[14]}$, as follows: F0, no fibrosis; F1, portal fibrosis without septa; F2, portal fibrosis with rare septa; F3, numerous septa without cirrhosis; F4, cirrhosis. F $\geq 2$ was considered as significant fibrosis and $\mathrm{F} \geq 3$ as advanced fibrosis.

\section{Serum Fibrosis Tests}

The aspartate aminotransferase-to-platelet ratio index (APRI) and the fibrosis-4 index (FIB-4) were calculated as (aspartate aminotransferase [international units/liter]/upper normal limit $\times 100$ /platelet counts $\left[\times 10^{9} /\right.$ liter] $)$ and (age [years] $\times$ aspartate aminotransferase [international units/liter]) $/($ platelet counts $\left[\times 10^{9} /\right.$ liter $] \times$ alanine aminotransferase [international units/liter $\left.]^{1 / 2}\right)^{[15,16]}$, respectively. These indices were calculated by using the results of laboratory tests performed within 26 days \pm 13 (range, 376 days) from obtaining results of pathologic examination of the liver. 


\section{CT Image Acquisition}

Contrast-enhanced CT scans were acquired in the axial plane with 0.75-1.5-mm-thick sections and a 0.751.5-mm reconstruction interval. Image acquisition parameters are detailed in Appendix E1.

\section{Radiomic Feature Extraction and Selection}

One reader (S.N.T., with 7 years of clinical experience in abdominal radiology) selected regions of interest (ROIs) in the liver of all patients. ROls for the liver were delineated along the margin of the right hepatic lobe, at the level of the right portal vein, by excluding large hepatic vessels and masses on non-contrast (mean area of ROls, $48 \mathrm{~cm}^{2} \pm 16$; range, $17-108 \mathrm{~cm}^{2}$ ), arterial (mean area of ROls, $48 \mathrm{~cm}^{2} \pm 18$; range, 16$108 \mathrm{~cm}^{2}$ ) and portal (mean area of ROls, $51 \mathrm{~cm}^{2} \pm 18$; range, $16-109 \mathrm{~cm}^{2}$ ) venous phases CT images by using 3D slicer (version 4.11.1; http://www.slicer.org). To explore the stability of each feature, 30 patients were randomly chosen; reader 1 repeated image segmentation twice and reader 2 independently performed segmentation to evaluate the intra- and interobserver reproducibility. The reproducibility was quantified by the intraclass correlation coefficient (ICC).

Image preprocessing and feature extraction were performed using the open-source Pyradiomics package (version 2.2.0: http://www.radiomics.io/pyradiomics.html). The voxel spacing was standardized with the size of $1 \times 1 \times 1 \mathrm{~mm}$ and voxel intensity values were discretized with a bin width of $25 \mathrm{HU}$ to reduce the interference of image noise and normalize intensities ${ }^{[17]}$, respectively. We extracted 837 radiomic features (18 first-order statistics, 75 textural features and 744 wavelet transformations) from each twodimensional segmentation, giving a total of 2511 for every phase CT images (non-contrast, arterial and portal venous phases). The $z$-scores was used to standardize values of features and the mean and standard deviation determined in the training cohort were applied in the validation cohort.

A three-step procedure was followed to select significant radiomic features. First, the reliability of each feature was qualified by using ICC and features with ICC more than 0.9 were kept for further analysis ${ }^{[18]}$. Second, irrelevant features that weakly correlated with fibrosis stage were removed; the correlation between each radiomic feature and metavir fibrosis stage was evaluated by using the Kendall correlation coefficient. Features with correlation coefficients less than 0.15 were eliminated. The final step in feature selection was performed by using the least absolute shrinkage and selection operator (LASSO) logistic regression algorithm with penalty parameter tuning conducted by 10 -fold cross-validation ${ }^{[19]}$, between stages F0-F2 and F3-F4, and features with nonzero coefficients were considered independently related to fibrosis stage.

\section{Clinical factors selection}

We devised a three-step procedure for selection of clinical factors. First, we used kendall correlation analysis to screen out factors with significant correlation (kendall correlation analysis, $P<0.05$ ). Second, forward conditional logistic multivariable analysis was used to select factors for the discrimination between stages F0-F2 and F3-F4 (input and output $P$ value: 0.05 and 0.1 , respectively). Third, a function 
on the basis of the variance inflation factor (VIF) was conducted to check for the collinearity of variables included in the regression equations ${ }^{[20]}$. Variables with VIF greater than 10 (indicating multicollinearity) were excluded.

\section{Model Establishment and validation}

The radiomics signature for the prediction of fibrosis (R-score) was created using support vector machine (SVM) as a multi classification to distinguish among stages F0, F1, F2, F3 and F4. SVM was performed using "e1071" package (https://CRAN.R-project.org/package=e1071) on R software (version 3.6.1, http://www.r-project.org).

Multivariate linear regression analysis was performed to establish a final model based on radiomics signature and clinical factors for the prediction of fibrosis (R-fibrosis). The performance of models was tested in the independent validation cohort by using the equation derived from the training cohort.

\section{Statistical analysis}

Categorical and continuous variables were compared by using $\chi^{2}$ test and the Mann-Whitney $U$ test, respectively. The correlation between results calculated from models and pathologic liver fibrosis stage was evaluated by using the spearman correlation analysis. Performance of models for staging liver fibrosis was evaluated by using receiver operating characteristics (ROC) curve analysis, area under the curve (AUC) value and the Obuchowski index, a multinomial version of ROC curve analysis adapted for ordinal references such as metavir staging of liver fibrosis ${ }^{[21]}$. The Obuchowski index is a weighted average of the areas under the curve obtained for all possible pairs of fibrosis stages to be differentiated and it estimates the probability that a test will correctly rank two randomly chosen patients with different stages of fibrosis. The optimal thresholds of models were determined using the ROC analysis by maximizing the Youden index. Delong nonparameteric approach was used to compare AUC values ${ }^{[22]}$. Calibration curves were plotted to evaluate the calibration of the established model, accompanied by the Hosmer-Lemeshow test. Additionally, a decision curve analysis (DCA) was performed to assess the

clinical usefulness and net benefits of the developed radiomics models ${ }^{[23]}$. A two-sided $P$ value less than .05 was indicative of a statistically significant difference.

\section{Results}

\section{Characteristics of the study cohorts}

The baseline characteristics of all patients are summarized in Table 1. There were no significant differences in clinical and pathological characteristics between the training and validation cohorts. No differences were found in rates of significant fibrosis (Training: $69.0 \%, 229$ of 332; Validation: 78.4\%, 87 of $111 ; P=.06$ ), serious fibrosis (Training: $56.9 \%, 189$ of 332; Validation: $56.8 \%, 63$ of $111 ; P=.97$ ) and cirrhosis (Training: $40.1 \%, 133$ of 332; Validation: $38.8 \%, 43$ of $111 ; P=.81$ ) between the two cohorts. Results of APRI and FIB-4 were similar ( $P>.05$ for both) between the two study cohorts. 


\section{Fibrosis-related clinical factors}

In the training cohort, platelet (PLT) count, glutamyl transpeptidase (GGT), albumin (ALB), albumin to globulin ratio $(A / G)$ and total cholesterol (TC) were identified as independent fibrosis predictors by the multivariable logistic regression analysis (Table 2). The VIF of TC was 10.7 (over 10), indicating the collinearity, in which the variable should be excluded. According to the Kendall correlation coefficient of GGT and PLT ( 0.15 and -0.292 , respectively), the GGT to PLT ratio was involved in the prediction model. 
Table 2

Clinical characteristics of the training cohort related to fibrosis

\section{Kendall correlation analysis Multivariable analysis Collinearity Statistics}

\begin{tabular}{|lllllll|}
\hline Variables & Coefficient & P value & b coefficient & P value & Tolerance & VIF \\
\hline Age (years) & -0.042 & .33 & NA & NA & NA & NA \\
\hline Sex (male, female) & 0.087 & .10 & NA & NA & NA & NA \\
\hline RBC (109/L) & -0.107 & .01 & NA & .53 & 0.284 & 3.52 \\
\hline PLT (109/L) & -0.292 & $<.001$ & -0.012 & $<.001$ & 0.699 & 1.43 \\
\hline Hb (g/L) & -0.106 & .02 & NA & .66 & 0.276 & 3.63 \\
\hline ALT (U/L) & 0.089 & .04 & NA & .54 & 0.123 & 8.10 \\
\hline AST (U/L) & 0.164 & $<.001$ & NA & .98 & 0.115 & 8.68 \\
\hline ALP (U/L) & 0.147 & .001 & NA & .81 & 0.202 & 4.95 \\
\hline GGT (U/L) & 0.150 & .001 & 0.007 & .004 & 0.469 & 2.13 \\
\hline LDH (U/L) & 0.045 & .298 & NA & NA & NA & NA \\
\hline TB (umol/L) & 0.000 & .99 & NA & NA & NA & NA \\
\hline CB (umol/L) & 0.169 & $<.001$ & NA & .23 & 0.104 & 9.59 \\
\hline ALB (g/L) & -0.117 & .007 & 0.139 & .002 & 0.491 & 2.04 \\
\hline GLOB (g/L) & 0.081 & .06 & NA & NA & NA & NA \\
\hline A/G & -0.104 & .02 & -1.668 & $<.001$ & 0.693 & 1.44 \\
\hline TBA (umol/L) & 0.213 & $<.001$ & NA & .22 & 0.528 & 1.89 \\
\hline LAP (U/L) & 0.056 & .20 & NA & NA & NA & NA \\
\hline TC (mmol/L) & -0.184 & $<.001$ & -0.399 & .02 & 0.093 & 10.70 \\
\hline HDL-C (mmol/L) & 0.009 & .83 & NA & NA & NA & NA \\
\hline LDL-C (mmol/L) & -0.171 & $<.001$ & NA & .20 & 0.045 & 22.03 \\
\hline
\end{tabular}

Note. $--b$ coefficients are from multivariable logistic regression. Clinical variables found to be significantly related to cirrhosis through spearman correlation analysis entered into forward conditional logistic multivariate analysis. ALB= albumin, ALP= alkaline phosphatase, ALT= alanine aminotransferase, Apo A1 = apolipoprotein A1, Apo B= apolipoprotein B, AST= aspartate aminotransferase, $A / G=$ albumin to globulin ratio, $C B=$ conjugated bilirubin, $C R P=C$ reactive protein, GGT = glutamyl transpeptidase, $\mathrm{GLOB}=$ globulin, $\mathrm{Hb}=$ hemoglobin, $\mathrm{HDL}-\mathrm{C}=$ high density lipoprotein cholesterol, INR= international normalized ratio, LAP= leucine arylamidase, LDH= lactate dehydrogenase, $L D L-C=$ low density lipoprotein cholesterol; $P L T=$ blood platelet, $P T=$ prothrombin time, $\mathrm{RBC}=$ red blood cell, $\mathrm{TB}=$ serum total bilirubin, $\mathrm{TBA}=$ total bile acid, $\mathrm{TC}=$ total cholesterol, $\mathrm{VIF}=$ variance inflation factor. 


\begin{tabular}{|c|c|c|c|c|c|c|}
\hline \multirow[b]{2}{*}{ Apo A1 (g/L) } & \multicolumn{2}{|c|}{ Kendall correlation analysis } & \multicolumn{2}{|c|}{ Multivariable analysis } & \multicolumn{2}{|c|}{ Collinearity Statistics } \\
\hline & 0.010 & .83 & NA & NA & NA & NA \\
\hline Apo B (g/L) & -0.168 & $<.001$ & NA & .63 & 0.087 & 11.48 \\
\hline CRP (mg/L) & 0.101 & .02 & NA & .49 & 0.512 & 1.95 \\
\hline PT (s) & 0.189 & $<.001$ & NA & .40 & 0.017 & 59.27 \\
\hline INR & 0.210 & $<.001$ & NA & .35 & 0.017 & 60.28 \\
\hline \multicolumn{7}{|c|}{ 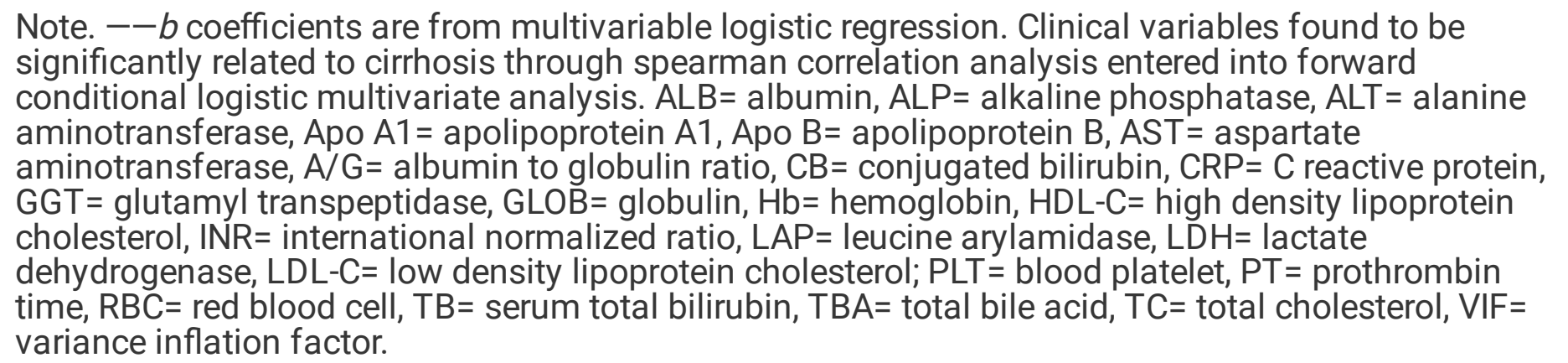 } \\
\hline
\end{tabular}

\section{Radiomic feature selection and signature construction}

Among 2084 radiomic features with high stability, 320 features with significant correlations to fibrosis stage were identified. And then, 21 independent features with nonzero coefficients were finally selected by the LASSO logistic regression (Figure 3). A radiomic signature was constructed using SVM algorithm. The type of SVM was "eps-classification", of which the kernel function was radial basis. The value of gamma and epsilon was 0.045 and 0.1, respectively. The total number of support vectors was 153 .

The R-score showed a positive correlation with liver fibrosis stage $(r=0.717, P<.001$; Figure 4$)$. In the training cohort, the R-score showed AUCs of 0.904 (95\% confidence interval [Cl]: 0.865, 0.942), 0.911 (95\% Cl: $0.880,0.943)$ and $0.844(95 \% \mathrm{Cl}: 0.800,0.889)$ for the diagnosis of significant fibrosis, advanced fibrosis and cirrhosis. The Obuchowski index, which indicates the overall accuracy of multiclass liver fibrosis staging, was 0.847 (95\% Cl: 0.797, 0.897) (Table 3). The R-score showed favorable discriminatory ability, with AUCs of 0.875 (95\% Cl: $0.781,0.969), 0.900$ ( $95 \% \mathrm{Cl}: 0.842,0.959)$ and 0.857 ( $95 \%$ Cl: $0.790,0.925)$, for significant fibrosis, advanced fibrosis and cirrhosis in the validation cohort (Table 4). 
Table 3

Diagnostic performance of models for staging liver fibrosis in the training cohort

\begin{tabular}{|c|c|c|}
\hline Parameter & R-score & R-fibrosis \\
\hline \multicolumn{3}{|c|}{ Significant fibrosis (F2-F4) } \\
\hline AUC & $0.904(0.865,0.942)$ & $0.905(0.867,0.943)$ \\
\hline Threshold & -0.258 & -0.403 \\
\hline Sensitivity (\%) & $92.1(87.9,95.3)$ & $94.8(91.0,97.3)$ \\
\hline Specificity (\%) & $76.7(67.3,84.5)$ & $74.8(65.2,82.8)$ \\
\hline Accuracy (\%) & $87.3(83.9,90.9)$ & $88.6(85.1,92.0)$ \\
\hline \multicolumn{3}{|c|}{ Advanced fibrosis (F3-F4) } \\
\hline AUC & $0.911(0.880,0.943)$ & $0.915(0.884,0.946)$ \\
\hline Threshold & 0.167 & 0.332 \\
\hline Sensitivity (\%) & $83.6(77.4,88.7)$ & $85.8(79.9,90.5)$ \\
\hline Specificity (\%) & $89.3(83.1,93.7)$ & $87.9(81.6,92.7)$ \\
\hline Accuracy (\%) & $86.1(82.4,89.9)$ & $86.7(83.1,90.4)$ \\
\hline \multicolumn{3}{|l|}{ Cirrhosis (F4) } \\
\hline AUC & $0.844(0.800,0.889)$ & $0.857(0.814,0.899)$ \\
\hline Threshold & 0.503 & 0.950 \\
\hline Sensitivity (\%) & $60.7(50.8,70.0)$ & $65.4(55.6,74.4)$ \\
\hline Specificity (\%) & $95.6(92.0,97.8)$ & $90.2(85.6,93.8)$ \\
\hline Accuracy (\%) & $84.3(80.4,88.3)$ & $82.2(78.1,86.4)$ \\
\hline Obuchowski index & $0.847(0.797,0.897)$ & $0.852(0.807,0.898)$ \\
\hline
\end{tabular}


Table 4

Areas under the curve and Obuchowski indexes of R-score and serum fibrosis tests for staging liver fibrosis in the validation cohort

\begin{tabular}{|c|c|c|c|c|}
\hline Parameter & R-score & R-fibrosis & APRI & FIB-4 \\
\hline $\begin{array}{l}\text { Significant fibrosis } \\
\text { (F2-F4) }\end{array}$ & $\begin{array}{l}0.875(0.781 \\
0.969)\end{array}$ & $\begin{array}{l}0.901(0.818 \\
0.984)\end{array}$ & $\begin{array}{l}0.692(0.581 \\
0.804){ }^{\star} \S\end{array}$ & $\begin{array}{l}0.713(0.619 \\
0.808) \star \S\end{array}$ \\
\hline $\begin{array}{l}\text { Advanced fibrosis } \\
\text { (F3-F4) }\end{array}$ & $\begin{array}{l}0.900(0.842 \\
0.959)\end{array}$ & $\begin{array}{l}0.883(0.822 \\
0.945)\end{array}$ & $\begin{array}{l}0.673(0.569 \\
0.776){ }^{\star} \S\end{array}$ & $\begin{array}{l}0.714(0.617 \\
0.811){ }^{\star} \S\end{array}$ \\
\hline Cirrhosis (F4) & $\begin{array}{l}0.857(0.790 \\
0.925)\end{array}$ & $\begin{array}{l}0.860(0.791 \\
0.930)\end{array}$ & $\begin{array}{l}0.653(0.533 \\
0.772){ }^{\star} \S\end{array}$ & $\begin{array}{l}0.701(0.581 \\
0.820){ }^{\star} \S\end{array}$ \\
\hline Obuchowski index & $\begin{array}{l}0.843(0.808 \\
0.877)\end{array}$ & $\begin{array}{l}0.846(0.812 \\
0.880)\end{array}$ & $\begin{array}{l}0.651(0.561 \\
0.742){ }^{\star} \S\end{array}$ & $\begin{array}{l}0.676(0.606 \\
0.780) \star \S\end{array}$ \\
\hline \multicolumn{5}{|c|}{$\begin{array}{l}\text { Note. }- \text { Data in parenthesis are } 95 \% \text { confidence intervals. APRI = aspartate aminotransferase-to- } \\
\text { platelet ratio index, AUC = area under the curve, FIB- } 4=\text { fibrosis- } 4 \text { index, R-score = radiomics signatur } \\
\text { for the prediction of fibrosis, R-fibrosis = final established model for the prediction of fibrosis. }\end{array}$} \\
\hline \multicolumn{5}{|c|}{ *Significantly different from the results of R-score $(P<.05)$. } \\
\hline
\end{tabular}

\section{Development and validation of the prediction model}

A prediction model (R-fibrosis) integrated the R-score, GGT to PLT ratio, A/G and ALB $(0,>40 \mathrm{~g} / \mathrm{L} ; 1, \leq 40$ $\mathrm{g} / \mathrm{L})$. The equation for R-fibrosis derived from the training cohort was: $\mathrm{R}$-fibrosis $=1.675 \times \mathrm{R}$-score + $0.107 \times$ GGT/PLT + 0.121×ALB×A/G.

The R-fibrosis showed a positive correlation with liver fibrosis stage $(r=0.743, P<.001$; Figure 4$)$. In the training cohort, AUCs of R-fibrosis for diagnosing significant fibrosis, advanced fibrosis and cirrhosis were 0.905 (95\% Cl: 0.867, 0.943), 0.915 (95\% Cl: 0.884, 0.946) and 0.915 (95\% Cl: 0.884, 0.946), respectively. And the Obuchowski index was 0.852 (95\% Cl: 0.807, 0.898).

Table 4 summarizes the diagnostic performance of R-fibrosis, R-score, APRI and FIB-4 in the validation cohort. The AUCs of R-fibrosis and R-score for aiding in diagnosis of significant fibrosis ( 0.901 and 0.875 , $95 \% \mathrm{Cl}:[0.818,0.984]$ and $[0.781,0.969])$, advanced fibrosis $(0.883$ and $0.900,95 \% \mathrm{Cl}:[0.822,0.945]$ and $[0.842,0.959])$ and cirrhosis $(0.860$ and $0.857,95 \% \mathrm{Cl}$ : $[0.791,0.930]$ and $[0.790,0.925])$ were higher than the AUCs for APRI (range, 0.653-0.692) and FIB-4 (range, 0.701-0.714) (Figure 5). By using thresholds determined in the training cohort, R-fibrosis and R-score had a sensitivity range of $70 \%-87 \%$, specificity range of $71 \%-97 \%$, and accuracy range of $82 \%-86 \%$ in diagnosing significant fibrosis, advanced fibrosis and cirrhosis in the validation cohort (Table 5). 
Table 5

Diagnostic performance of models for staging liver fibrosis in the validation cohort

\begin{tabular}{|c|c|c|c|c|c|}
\hline Parameter & Model & $\begin{array}{l}\text { Threshold } \\
\text { value* }\end{array}$ & Sensitivity (\%) & Specificity (\%) & Accuracy (\%) \\
\hline \multirow[t]{2}{*}{$\begin{array}{l}\text { Significant } \\
\text { fibrosis (F2-F4) }\end{array}$} & R-score & -0.258 & $\begin{array}{l}83.9(73 / 87) \\
{[76.0,91.8]}\end{array}$ & $\begin{array}{l}87.5(21 / 24) \\
{[73.2,99.9]}\end{array}$ & $\begin{array}{l}84.7(94 / 111) \\
{[77.9,91.5]}\end{array}$ \\
\hline & $\begin{array}{l}\text { R- } \\
\text { fibrosis }\end{array}$ & -0.403 & $\begin{array}{l}87.4(76 / 87) \\
{[80.2,94.5]}\end{array}$ & $\begin{array}{l}70.8(17 / 24) \\
{[51.2,90.4]}\end{array}$ & $\begin{array}{l}83.8(93 / 111) \\
{[76.8,90.7]}\end{array}$ \\
\hline \multirow[t]{2}{*}{$\begin{array}{l}\text { Advanced fibrosis } \\
\text { (F3-F4) }\end{array}$} & R-score & 0.167 & $\begin{array}{l}71.4(45 / 63) \\
{[60.0,82.9]}\end{array}$ & $\begin{array}{l}95.8(46 / 48) \\
{[90.0,99.9]}\end{array}$ & $\begin{array}{l}82.0(91 / 111) \\
{[74.7,89.2]}\end{array}$ \\
\hline & $\begin{array}{l}\text { R- } \\
\text { fibrosis }\end{array}$ & 0.332 & $\begin{array}{l}76.2(48 / 63) \\
{[65.4,87.0]}\end{array}$ & $\begin{array}{l}91.7(44 / 48) \\
{[83.6,99.8]}\end{array}$ & $\begin{array}{l}82.9(92 / 111) \\
{[75.8,90.0]}\end{array}$ \\
\hline \multirow[t]{2}{*}{ Cirrhosis (F4) } & R-score & 0.503 & $\begin{array}{l}69.8(30 / 43) \\
{[55.5,84.1]}\end{array}$ & $\begin{array}{l}91.2(62 / 68) \\
{[84.3,98.1]}\end{array}$ & $\begin{array}{l}82.9(92 / 111) \\
{[75.8,90.0]}\end{array}$ \\
\hline & $\begin{array}{l}\text { R- } \\
\text { fibrosis }\end{array}$ & 0.950 & $\begin{array}{l}79.1(34 / 43) \\
{[66.4,91.7]}\end{array}$ & $\begin{array}{l}89.7(61 / 68) \\
{[82.3,97.1]}\end{array}$ & $\begin{array}{l}85.6(95 / 111) \\
{[78.9,92.2]}\end{array}$ \\
\hline \multicolumn{6}{|c|}{$\begin{array}{l}\text { Note. -Data in parenthesis are numerator/denominator and data in brackets are } 95 \% \text { confidence } \\
\text { interval. }\end{array}$} \\
\hline
\end{tabular}

Calibration curves of the R-fibrosis and R-score demonstrated great agreement between predicted and actual significant fibrosis, advanced fibrosis and cirrhosis in the validation cohort (Figure 5). The HosmerLemeshow test yielded a $P$ value of $>.05$, suggesting no departure from the good fit. The decision curve analysis for the R-fibrosis, R-score, APRI and FIB-4 are presented in Figure 5. R-fibrosis and R-score provided higher net benefit compared with other models and simple strategies of all patients or no patients across the majority of the range of reasonable threshold probabilities in the validation cohort. No obvious differences were found in terms of clinical benefit between R-fibrosis and R-score.

\section{Discussion}

The aim of this study was to develop and validate radiomics-based models on contrast-enhanced CT radiomics for liver fibrosis. We concluded that radiomics analysis of contrast-enhanced CT allows for more accurate staging of liver fibrosis compared with other models. The R-fibrosis and R-score created by the training cohort data predicted the staging of liver fibrosis in the validation cohort with AUCs of 0.840.90 and accuracies of $82 \%-86 \%$. In agreement with our hypothesis, radiomics models (Obuchowski index, 0.84-0.85) outperformed custom serum indices (Obuchowski index, 0.65-0.68).

There are various less-invasive methods for staging liver fibrosis including serological markers and elastography. Ultrasound-based elastography (including transient elastography [TE] and two-dimensional shear wave elastography [2D-SWE]) and magnetic resonance elastography (MRE) are known to have 
great diagnostic performance for staging liver fibrosis ${ }^{[19,24,25]}$. However, elastography techniques are not widely used in China because of high prices and limited cost-effectiveness for general hospitals. HBV carriers are frequently suggested to receive annual contrast-enhanced CT or MRI in China. Our previous study developed a radiomics-based model at non-contrast CT for predicting cirrhosis ${ }^{[13]}$ and this study used contrast-enhanced CT for further investigation (significant \& advanced fibrosis).

Both contrast-enhanced CT and MRI are recommended by guidelines for early detection of liver tumor for patients with chronic liver diseases ${ }^{[7,26]}$, and many studies have focused on image data mining at MRI involving image findings and texture analysis ${ }^{[27-29]}$. Actually, CT is more readily available than MRI. Computer-aided visual assessment of liver or spleen volume and homogeneity on CT allowed for the detection of fibrosis stage but showed neglect of multiclass accuracy ${ }^{[10,30]}$. Moreover, none of them were validated in independent test data sets. A deep convolutional neural network (DCNN) system for staging liver fibrosis was developed by using portal venous phases CT images ${ }^{[31]}$. Unlike texture analysis, the DCNN system extracted and analyzed features from cropped and zoomed images. Diagnostic performance of the DCNN system is not greater than us (AUC range: 0.73-0.76), although there should be a head-to-head comparison for comparing these two methods.

The established radiomic signature (R-score) in this study included 4 first-order statistics and 17 textural features. As similar to previous studies ${ }^{[32,33]}$, most of $(90.5 \%, 19$ of 21$)$ these included features were processed by wavelet transform. 12 features were derived from non-contrast CT and others were from arterial (4) and portal (5) venous phases, of which the cause might be non-contrast CT can provide more stable features without effects of personal intake. The final model (R-fibrosis) included GGT/PLT, ALB and $A / G$ in addition to R-score. The predictive value of the GGT to PLT ratio for significant fibrosis and cirrhosis was confirmed by Lemoine et $\mathrm{al}^{[34]}$. and Lu et al ${ }^{[35]}$. ALB has been confirmed as an independent indicator of advanced liver fibrosis in patients with NAFLD ${ }^{[36]}$, and it can also significantly contribute to the index for staging liver fibrosis in patients with viral hepatitis ${ }^{[37,38]} . A / G$ was used as biomarkers in many cases such as tumor prognosis ${ }^{[39-41]}$ and chronic diseases ${ }^{[42-44]}$, but only one study involved $A / G$ into the fibrosis markers ${ }^{[45]}$. The specificity of $A / G$ for fibrosis might not so high, and thus we make it computable when ALB $\leq 40 \mathrm{~g} / \mathrm{L}$.

There were several limitations in our study. First, the limited population size and the unbalanced distribution of the patient population restricted the great establishment of the prediction model. Moreover, the retrospective study may introduce selection biases, and there were larger numbers of patients with advanced fibrosis (i.e. stages F3\&F4) than others (i.e. stages F0-F2). Second, the proposed radiomicsbased model was established using data obtained from a single center. Our model needed to be further validated by prospective multicenter studies with considerably large datasets. Third, image findings related to significant fibrosis (a nodular or irregular hepatic surface, parenchymal abnormalities, a blunt liver edge, intrahepatic morphological changes and clinical manifestations of portal hypertension) were not considered in this study. The main reason is that these image findings are frequently suggestive of 
cirrhosis $^{[46]}$. Finally, because elastography methods (TE or 2DSWE) did not performed for these patients, we were unable to compare the efficacy of our model with that of elastography for staging liver fibrosis.

In conclusion, we proposed a noninvasive and convenient radiomics-based model at contrast-enhanced CT images which allowed for accurate diagnosis of clinically significant liver fibrosis. Compared with our previous radiomics model based on non-contrast $\mathrm{CT}$ scans, R-fibrosis can additionally become as an update version for the prediction of significant and advanced fibrosis.

\section{Abbreviations}

CT, computed tomography; NAFLD, non-alcoholic fatty liver diseases; AASLD, American Association for the Study of Liver Diseases; HBV, hepatitis B virus; APRI, aspartate aminotransferase-to-platelet ratio index; FIB-4, fibrosis-4 index; ROI, region of interest; ICC, intraclass correlation coefficient; LASSO, least absolute shrinkage and selection operator; VIF, variance inflation factor; R-score, radiomics signature for the prediction of fibrosis; SVM, support vector machine; R-fibrosis, final model based on radiomics signature and clinical factors for the prediction of fibrosis; ROC, receiver operating characteristics; AUC, area under the curve; DCA, decision curve analysis; PLT, platelet; GGT, glutamyl transpeptidase; ALB, albumin; $A / G$, albumin to globulin ratio; $T C$, total cholesterol; $\mathrm{Cl}$, confidence interval; $T E$, transient elastography; 2D-SWE, two-dimensional shear wave elastography; MRE, magnetic resonance elastography; DCNN, deep convolutional neural network.

\section{Declarations}

\section{Acknowledgements:}

We would like to express our great appreciation to the R Development Core Team and contributors for $\mathrm{R}$ packages used in our study.

\section{Author contributions:}

Study Concept: Beicheng Sun and Jian He

Study Supervision: Yin Yin

Study Design: Jun Chen, Yin Yin, Jian He

Acquisition and governance of the data: Jincheng Wang, Shengnan Tang, Yingfan Mao, Jin Wu and Shanshan Xu

Statistical Analysis: Jincheng Wang, Ying-Fan Mao and Qi Yue

Data Interpretation: Jincheng Wang, Shanshan Xu and Jun Chen

Draft of Manuscript: Yin Yin 
Critical Revision of the Manuscript: Jincheng Wang, Yingfan Mao and Yin Yin

All authors performed data analysis and interpretation and read and approved the final manuscript.

\section{Funding}

This work was supported by grants from the National Natural Science Youth Foundation (Grant Number 81902415 to Y.Y.), Natural Science Youth Foundation of Jiangsu Province (Grant Number BK20190116 to Y.Y.) and Key Laboratory of Imaging Diagnosis and Minimally Invasive Interventional Research of Zhejiang Province (No. YXJR202002, to J.W).

\section{Availability of data and materials}

The data is not available because of patients' privacy.

\section{Ethics approval and consent to partipate}

This retrospective study was approved by the institutional review board of the Affiliated Drum Tower Hospital of Nanjing University Medical School. The requirement for written informed consent was waived due to its retrospective nature.

\section{Consent for publication}

Not applicable.

\section{Competing interests}

The authors declare that they have no competing interests.

\section{Author details}

1. Department of Hepatobiliary Surgery, The Affiliated Drum Tower Hospital of Nanjing University Medical School, Nanjing, China;

2. Department of Hepatobiliary Surgery of Drum Tower Clinical Medical College, Nanjing Medical University, Nanjing, China;

3. Department of Radiology, The Affiliated Drum Tower Hospital of Nanjing University Medical School, Nanjing, China;

4. Department of Pathology, The Affiliated Drum Tower Hospital of Nanjing University Medical School, Nanjing, China.

\section{References}

1. R. Bataller and D. A. Brenner, Liver fibrosis. J Clin Invest, 2005. 115(2): p. 209-18. 
2. Liver European Association for Study Of and Higado Asociacion Latinoamericana Para El Estudio Del, EASL-ALEH Clinical Practice Guidelines: Non-invasive tests for evaluation of liver disease severity and prognosis. J Hepatol, 2015. 63(1): p. 237-64.

3. J. Perrault, D. B. Mcgill, B. J. Ott, et al., Liver biopsy: complications in 1000 inpatients and outpatients. Gastroenterology, 1978. 74(1): p. 103-6.

4. C. P. Strassburg and M. P. Manns, Approaches to liver biopsy techniques-revisited. Semin Liver Dis, 2006. 26(4): p. 318-27.

5. B. Maharaj, R. J. Maharaj, W. P. Leary, et al., Sampling variability and its influence on the diagnostic yield of percutaneous needle biopsy of the liver. Lancet, 1986. 1(8480): p. 523-5.

6. A. Regev, M. Berho, L. J. Jeffers, et al., Sampling error and intraobserver variation in liver biopsy in patients with chronic HCV infection. Am J Gastroenterol, 2002. 97(10): p. 2614-8.

7. J. A. Marrero, L. M. Kulik, C. B. Sirlin, et al., Diagnosis, Staging, and Management of Hepatocellular Carcinoma: 2018 Practice Guidance by the American Association for the Study of Liver Diseases. Hepatology, 2018. 68(2): p. 723-750.

8. N. Daginawala, B. Li, K. Buch, et al., Using texture analyses of contrast enhanced CT to assess hepatic fibrosis. Eur J Radiol, 2016. 85(3): p. 511-7.

9. A. L. Simpson, L. B. Adams, P. J. Allen, et al., Texture analysis of preoperative CT images for prediction of postoperative hepatic insufficiency: a preliminary study. J Am Coll Surg, 2015. 220(3): p. 339-46.

10. M. Romero-Gomez, E. Gomez-Gonzalez, A. Madrazo, et al., Optical analysis of computed tomography images of the liver predicts fibrosis stage and distribution in chronic hepatitis $C$. Hepatology, 2008. 47(3): p. 810-6.

11. R. J. Gillies, P. E. Kinahan, and H. Hricak, Radiomics: Images Are More than Pictures, They Are Data. Radiology. 278(2): p. 563-77.

12. H. J. Aerts, E. R. Velazquez, R. T. Leijenaar, et al., Decoding tumour phenotype by noninvasive imaging using a quantitative radiomics approach. Nat Commun, 2014. 5: p. 4006.

13. J. C. Wang, R. Fu, X. W. Tao, et al., A radiomics-based model on non-contrast CT for predicting cirrhosis: make the most of image data. Biomark Res, 2020. 8: p. 47.

14. P. Bedossa and T. Poynard, An algorithm for the grading of activity in chronic hepatitis $C$. The METAVIR Cooperative Study Group. Hepatology, 1996. 24(2): p. 289-93. 
15. C. T. Wai, J. K. Greenson, R. J. Fontana, et al., A simple noninvasive index can predict both significant fibrosis and cirrhosis in patients with chronic hepatitis C. Hepatology, 2003. 38(2): p. 518-26.

16. A. Vallet-Pichard, V. Mallet, B. Nalpas, et al., FIB-4: an inexpensive and accurate marker of fibrosis in HCV infection. comparison with liver biopsy and fibrotest. Hepatology, 2007. 46(1): p. 32-6.

17. J. J. M. Van Griethuysen, A. Fedorov, C. Parmar, et al., Computational Radiomics System to Decode the Radiographic Phenotype. Cancer Res, 2017. 77(21): p. e104-e107.

18. P. E. Shrout and J. L. Fleiss, Intraclass correlations: uses in assessing rater reliability. Psychol Bull, 1979. 86(2): p. 420-8.

19. M. Friedrich-Rust, M. F. Ong, S. Martens, et al., Performance of transient elastography for the staging of liver fibrosis: a meta-analysis. Gastroenterology, 2008. 134(4): p. 960-74.

20. Rm O'brien, A Caution Regarding Rules of Thumb for Variance Inflation Factors. Quality \& Quantity, 2007. 41(5): p. 673-690.

21. J. Lambert, P. Halfon, G. Penaranda, et al., How to measure the diagnostic accuracy of noninvasive liver fibrosis indices: the area under the ROC curve revisited. Clin Chem, 2008. 54(8): p. 1372-8.

22. E. R. Delong, D. M. Delong, and D. L. Clarke-Pearson, Comparing the areas under two or more correlated receiver operating characteristic curves: a nonparametric approach. Biometrics, 1988. 44(3): $p$. 837-45.

23. E. W. Steyerberg and A. J. Vickers, Decision curve analysis: a discussion. Med Decis Making, 2008. 28(1): p. 146-9.

24. E. Herrmann, V. De Ledinghen, C. Cassinotto, et al., Assessment of biopsy-proven liver fibrosis by two-dimensional shear wave elastography: An individual patient data-based meta-analysis. Hepatology, 2018. 67(1): p. 260-272.

25. S. Singh, S. K. Venkatesh, Z. Wang, et al., Diagnostic performance of magnetic resonance elastography in staging liver fibrosis: a systematic review and meta-analysis of individual participant data. Clin Gastroenterol Hepatol, 2015. 13(3): p. 440-451 e6.

26. Easloffice Easloffice Eu European Association for the Study of the Liver. Electronic Address and Liver European Association for the Study of The, EASL Clinical Practice Guidelines: Management of hepatocellular carcinoma. J Hepatol, 2018. 69(1): p. 182-236.

27. H. Watanabe, M. Kanematsu, S. Goshima, et al., Staging hepatic fibrosis: comparison of gadoxetate disodium-enhanced and diffusion-weighted MR imaging-preliminary observations. Radiology, 2011. 259(1): p. 142-50. 
28. L. Petitclerc, G. Sebastiani, G. Gilbert, et al., Liver fibrosis: Review of current imaging and MRI quantification techniques. J Magn Reson Imaging, 2017. 45(5): p. 1276-1295.

29. H. J. Park, S. S. Lee, B. Park, et al., Radiomics Analysis of Gadoxetic Acid-enhanced MRI for Staging Liver Fibrosis. Radiology, 2019. 290(2): p. 380-387.

30. J. H. Son, S. S. Lee, Y. Lee, et al., Assessment of liver fibrosis severity using computed tomographybased liver and spleen volumetric indices in patients with chronic liver disease. Eur Radiol, 2020. 30(6): p. 3486-3496.

31. K. Yasaka, H. Akai, A. Kunimatsu, et al., Deep learning for staging liver fibrosis on CT: a pilot study. Eur Radiol, 2018. 28(11): p. 4578-4585.

32. G. W. Ji, Y. D. Zhang, H. Zhang, et al., Biliary Tract Cancer at CT: A Radiomics-based Model to Predict Lymph Node Metastasis and Survival Outcomes. Radiology, 2019. 290(1): p. 90-98.

33. X. Xu, H. L. Zhang, Q. P. Liu, et al., Radiomic analysis of contrast-enhanced CT predicts microvascular invasion and outcome in hepatocellular carcinoma. J Hepatol, 2019. 70(6): p. 1133-1144.

34. M. Lemoine, Y. Shimakawa, S. Nayagam, et al., The gamma-glutamyl transpeptidase to platelet ratio (GPR) predicts significant liver fibrosis and cirrhosis in patients with chronic HBV infection in West Africa. Gut, 2016. 65(8): p. 1369-76.

35. X. J. Lu, X. H. Li, Z. X. Yuan, et al., Assessment of liver fibrosis with the gamma-glutamyl transpeptidase to platelet ratio: a multicentre validation in patients with HBV infection. Gut, 2018. 67(10): p. 1903-1904.

36. P. Angulo, J. M. Hui, G. Marchesini, et al., The NAFLD fibrosis score: a noninvasive system that identifies liver fibrosis in patients with NAFLD. Hepatology, 2007. 45(4): p. 846-54.

37. K. Fujita, T. Nomura, A. Morishita, et al., Albumin-Bilirubin Score Differentiates Liver Fibrosis Stage and Hepatocellular Carcinoma Incidence in Chronic Hepatitis B Virus Infection: A Retrospective Cohort Study. Am J Trop Med Hyg, 2019. 101(1): p. 220-225.

38. K. Fujita, K. Oura, H. Yoneyama, et al., Albumin-bilirubin score indicates liver fibrosis staging and prognosis in patients with chronic hepatitis C. Hepatol Res, 2019. 49(7): p. 731-742.

39. S. Omura, S. Taguchi, S. Miyagawa, et al., Prognostic significance of the albumin-to-globulin ratio for upper tract urothelial carcinoma. BMC Urol, 2020. 20(1): p. 133.

40. G. Y. Lv, L. An, X. D. Sun, et al., Pretreatment albumin to globulin ratio can serve as a prognostic marker in human cancers: a meta-analysis. Clin Chim Acta, 2018. 476: p. 81-91. 
41. C. C. Zhang, C. W. Zhang, H. Xing, et al., Preoperative Inversed Albumin-to-Globulin Ratio Predicts Worse Oncologic Prognosis Following Curative Hepatectomy for Hepatocellular Carcinoma. Cancer Manag Res, 2020. 12: p. 9929-9939.

42. J. Park, H. J. Kim, J. Kim, et al., Predictive value of serum albumin-to-globulin ratio for incident chronic kidney disease: A 12-year community-based prospective study. PLoS One, 2020. 15(9): p. e0238421.

43. X. R. Liu, Y. Y. Qi, Y. F. Zhao, et al., Albumin-to-globulin ratio (AGR) as a potential marker of predicting lupus nephritis in Chinese patients with systemic lupus erythematosus. Lupus, 2021. 30(3): p. 412-420.

44. J. T. Niedziela, B. Hudzik, B. Szygula-Jurkiewicz, et al., Albumin-to-globulin ratio as an independent predictor of mortality in chronic heart failure. Biomark Med, 2018. 12(7): p. 749-757.

45. G. Feng, K. I. Zheng, Y. Y. Li, et al., Machine learning algorithm outperforms fibrosis markers in predicting significant fibrosis in biopsy-confirmed NAFLD. J Hepatobiliary Pancreat Sci, 2021. 28(7): p. 593-603.

46. A. Huber, L. Ebner, J. T. Heverhagen, et al., State-of-the-art imaging of liver fibrosis and cirrhosis: A comprehensive review of current applications and future perspectives. Eur J Radiol Open, 2015. 2: p. 90100.

\section{Figures}




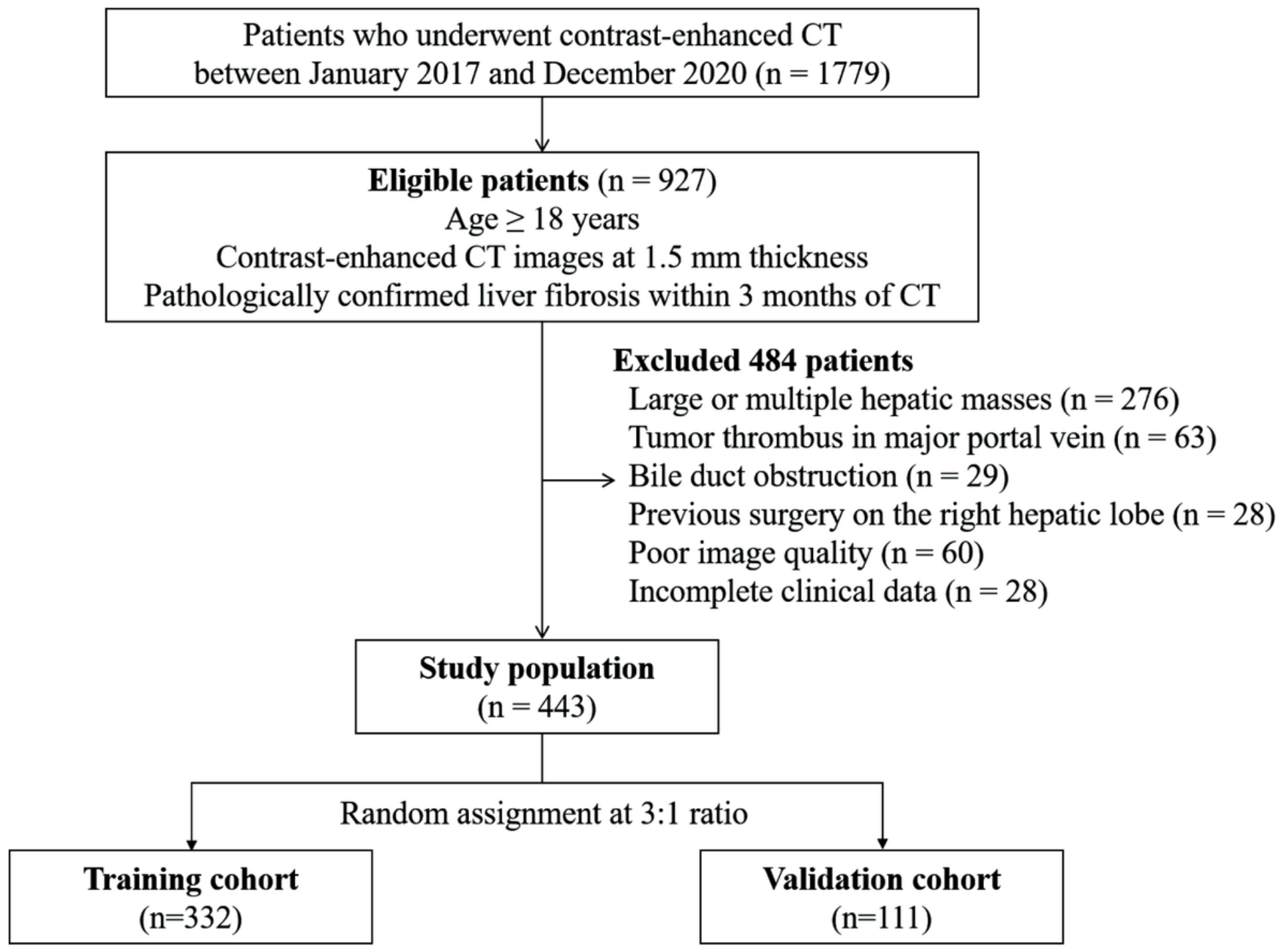

Figure 1

Patient selection flow chart. CT = computed tomography. 
Non-contrast phase
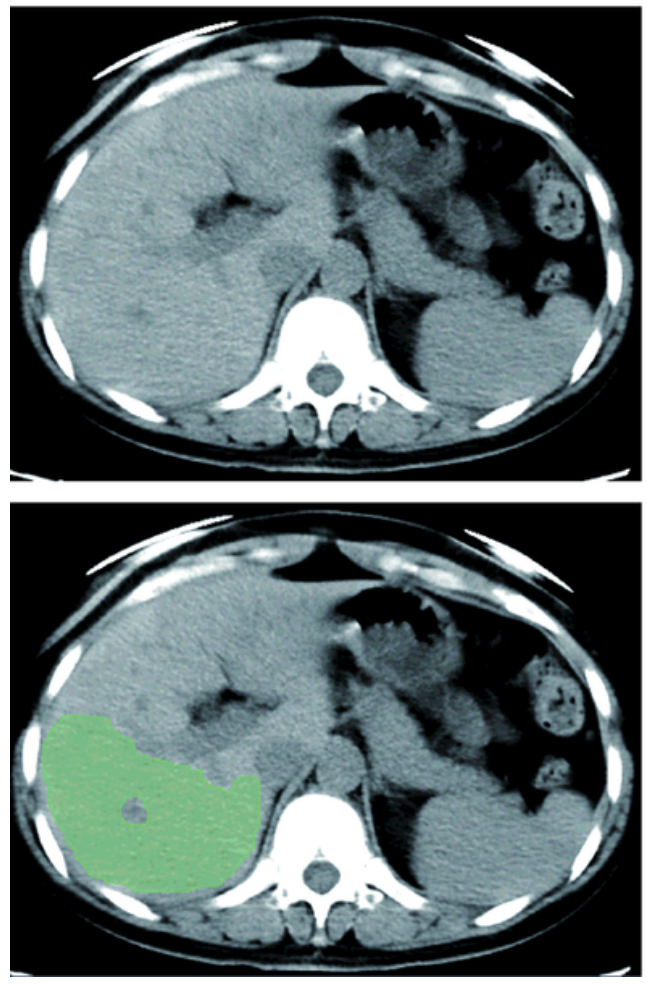

Arterial venous phase
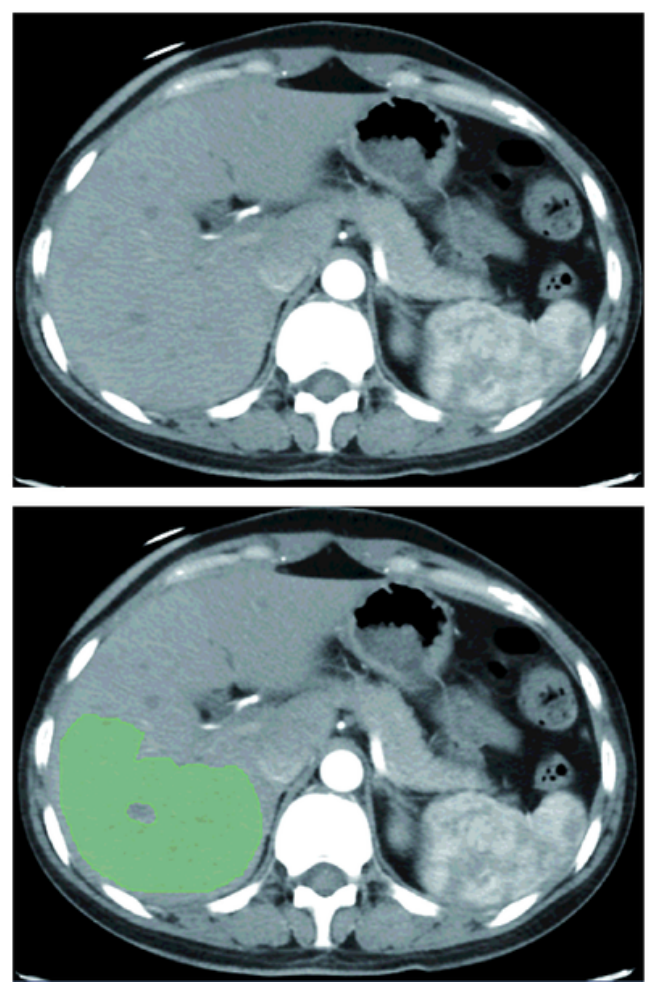

\section{Portal venous phase}
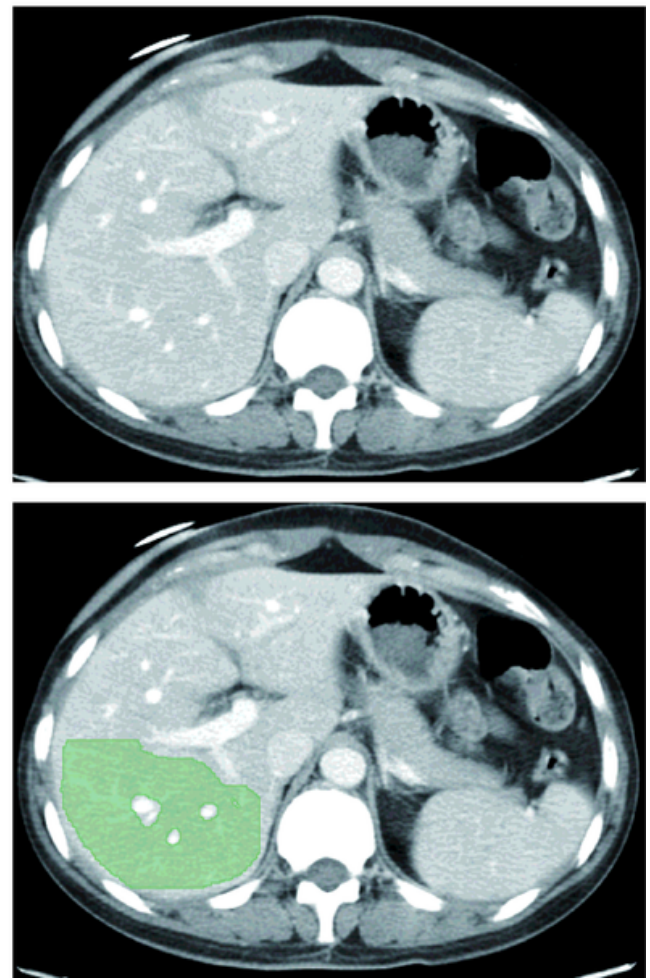

Figure 2

ROls for the liver at contrast-enhanced CT. ROls were delineated along the margin of the right hepatic lobe, at the level of the right portal vein, by excluding large hepatic vessels and masses on non-contrast, arterial and portal venous phases CT images. CT = computed tomography, ROI = region of interest.

A

$\begin{array}{llllllllllllllllll}89 & 86 & 87 & 81 & 81 & 69 & 67 & 59 & 56 & 47 & 36 & 27 & 20 & 16 & 13 & 8 & 3 & 1\end{array}$

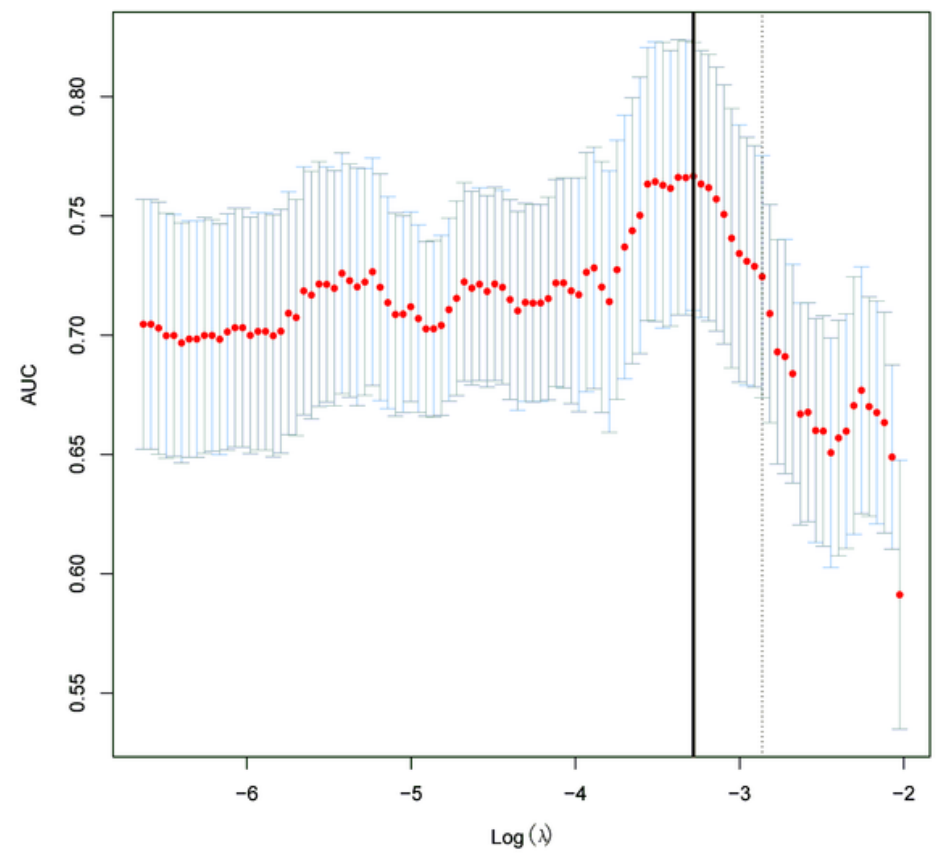

B

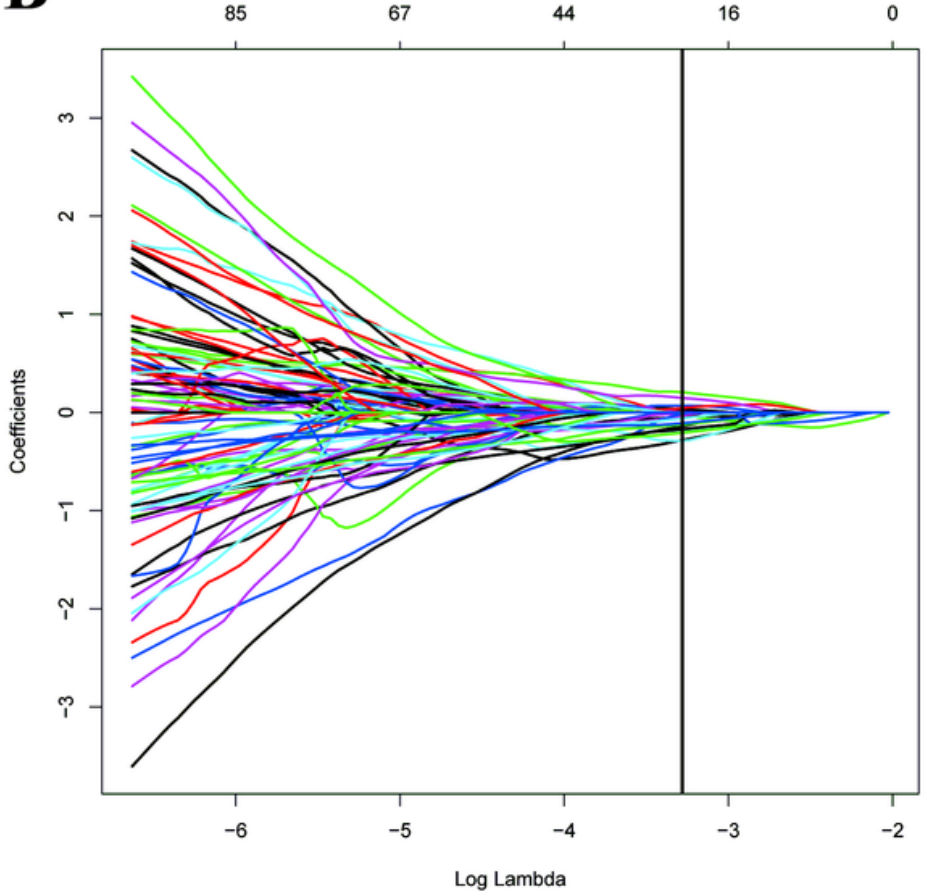

Figure 3 
Selections of radiomic features using the LASSO regression. A Optimal $\lambda$ value was determined by the LASSO model using 10 -fold cross-validation via minimum criteria. The AUC curve was plotted versus $\log (\lambda)$. Dotted vertical lines were drawn at the optimal values by using the minimum criteria and the 1 standard error of the minimum criteria (the 1 - standard error criteria). The optimal $\lambda$ value of 0.0376 was chosen. B LASSO coefficient profiles of the 320 selected features is presented. AUC $=$ area under the curve. LASSO = least absolute shrinkage and selection operator.
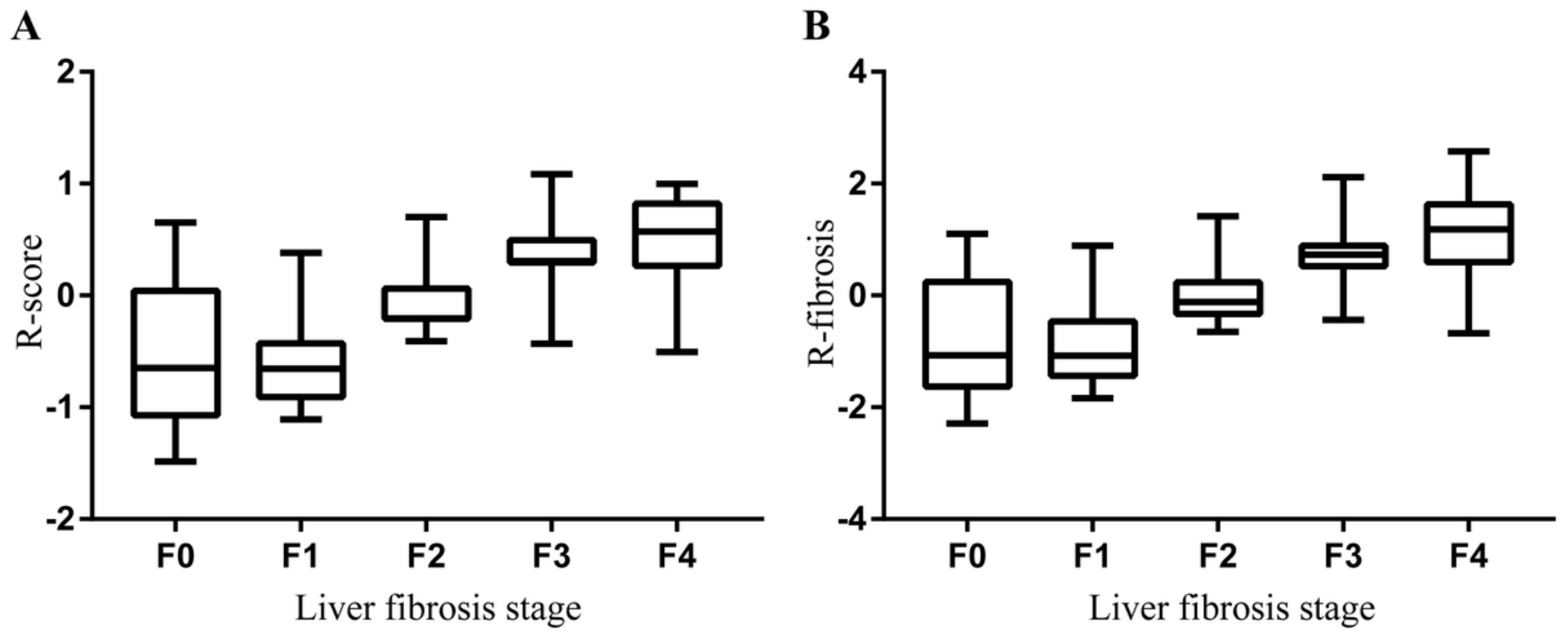

Figure 4

Box-and-whisker plot of the R-score and R-fibrosis for each pathologic liver fibrosis stage in the training cohort. Boxes, thick horizontal bars within the boxes, and whiskers represent interquartile ranges (IQRs), medians and $1.5 \times I Q R$, respectively. Both R-score and R-fibrosis have positive correlations with liver fibrosis stage $(r>0.7, P<.001$ for both). 


\section{A F0-F1 vs. F2-F4}

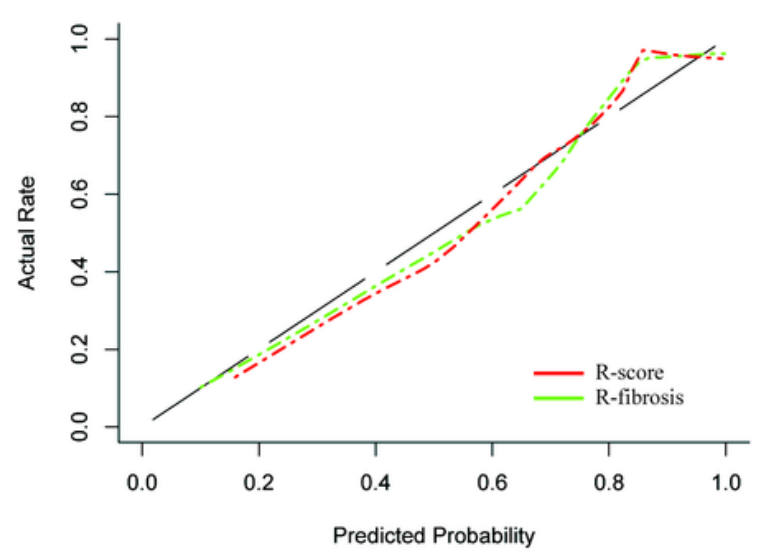

\section{B F0-F2 vs. F3-F4}

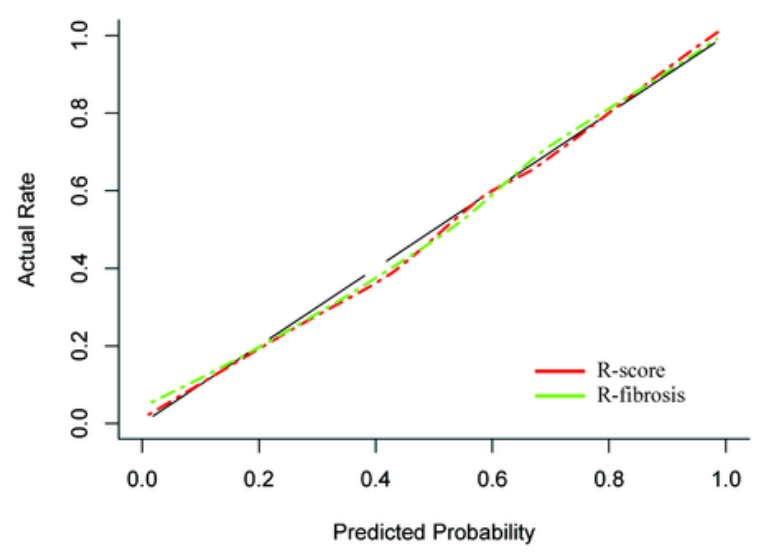

\section{F0-F3 vs. F4}

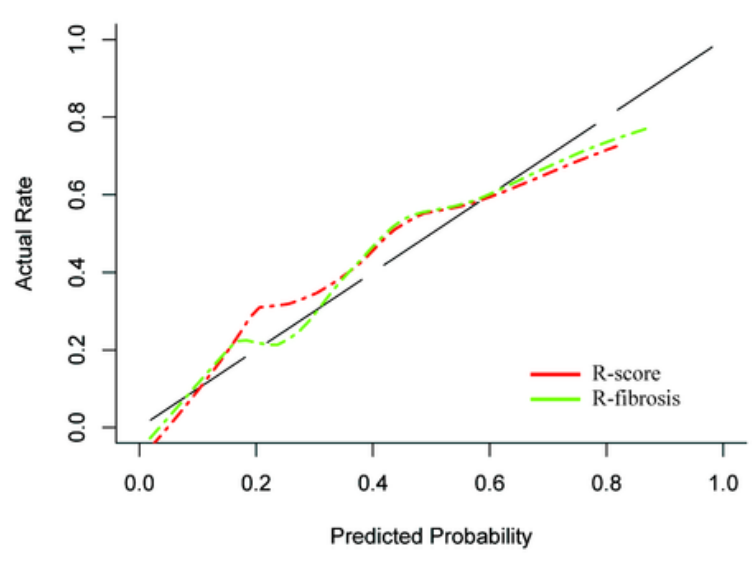

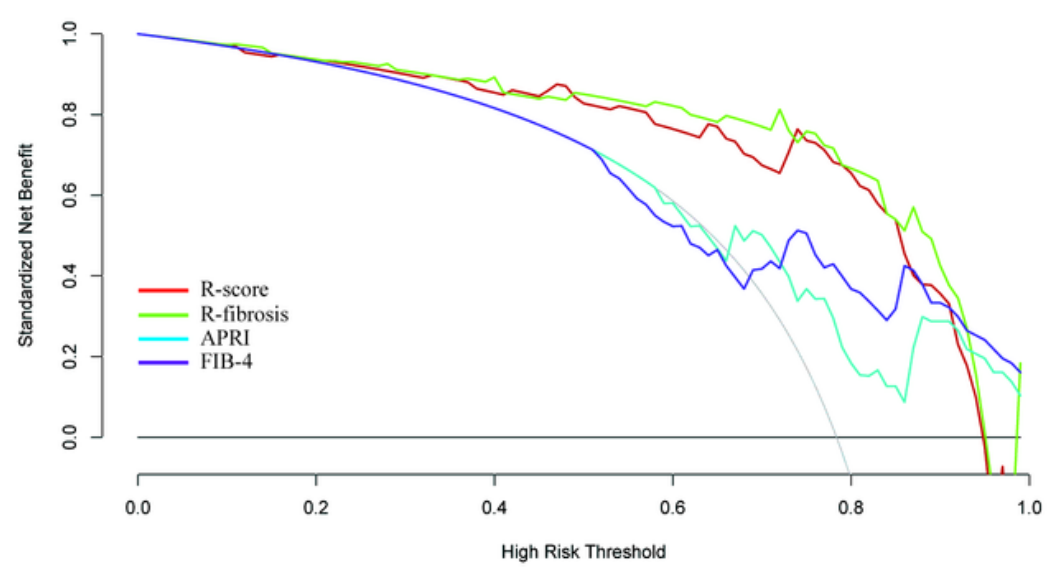
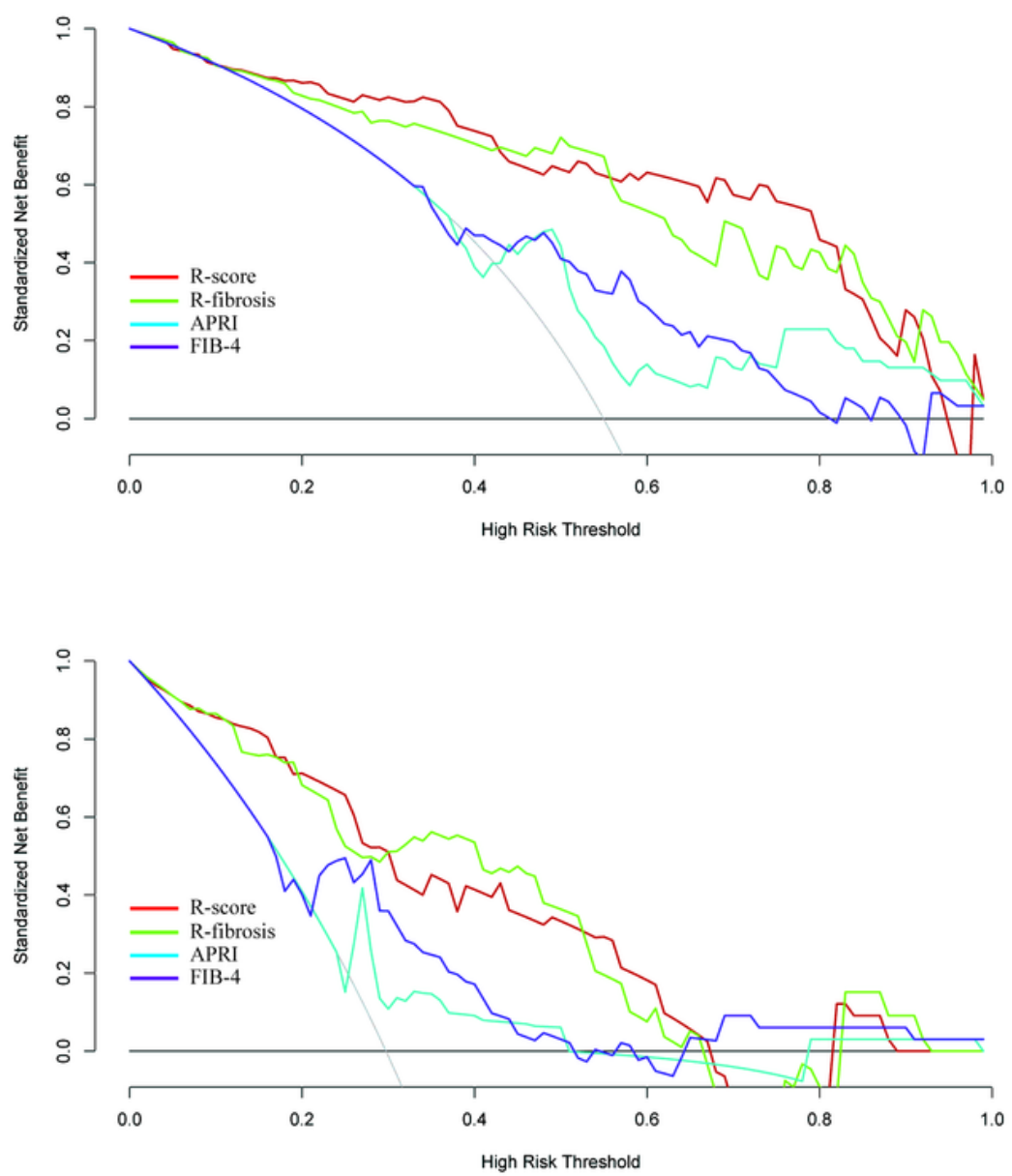

\section{Figure 5}

Calibration curves (left) and decision curve analysis (right) for each model in the validation dataset. Rscore and R-fibrosis were established due to the training cohort and validated for the prediction of significant fibrosis (A), advanced fibrosis (B) and cirrhosis (C). In decision curve analysis, the y-axis measures the net benefit, which was calculated by summing the benefits (true-positive results) and 
subtracting the harms (false-positive results), weighting the latter by a factor related to the relative harm of an undetected fibrosis status compared with the harm of unnecessary treatment.

\section{Supplementary Files}

This is a list of supplementary files associated with this preprint. Click to download.

- Supplementarymaterial.docx 